Д.В. Гринев, Д.С. Бойко, М.А. Голуб

Харьковский национальный экономический университет имени Семена Кузнеца, Харьков

\title{
ИСПОЛЬЗОВАНИЕ ВЕБ-САЙТОВ И СОЦИАЛЬНЫХ СЕТЕЙ В ЭЛЕКТРОННОЙ КОММЕРЦИИ
}

В статье рассмотрены основные подходы процесса прогнозирования поведения посетителя вебсайта. Приведены примеры существующих методик и методов прогнозирования, представлены выводы относительно необходимости анализа существующих и построения новых моделей поведения, а также составления прогнозов с помощью этих моделей в процессе ведения бизнеса. Также описаны методы использования сочиальных сетей для увеличения числа клиентов финансовых учреждений, повышения удовлетворенности продуктами банка и роста их конкурентоспособности. Определяется подход к стратегии использования сочиальных медиа в банковской сфере.

Ключевые слова: электронная коммеричия, модели поведения, социальная сеть, банк, маркетинг.

\section{Введение}

Стремительное развитие информационных технологий и такого феномена как Интернет в частности - кардинально изменило представление об экономических ценностях. Благодаря возможности вести бизнес с помощью информационных технологий, появилось такое понятие как «электронная коммерция». Общепринятого определения понятия «электронная коммерция» не существует. Однако, как правило, оно применяется в отношении "распространения маркетинга, продажи либо доставки товаров и услуг электронными средствами" [1].

Возможность совершать сделки между покупателем и поставщиком услуг является одной из ключевых для успешного ведения бизнеса. Только в 2016 годы объемы продаж с помощью электронной коммерции в США выросли на $15.6 \%$ (рис. 1), схожие показатели верны и для стран ЕС [2].

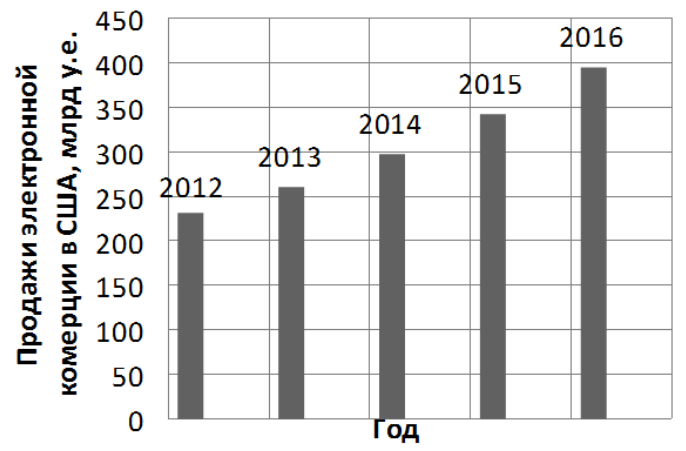

Рис. 1. Сумма продаж с помощью электронной коммерции в США

Электронная коммерция, также как и традиционные методы ведения бизнеса, состоит в проведении сделки между покупателем и продавцом. В данном случае продавцом будет являться веб-сайт, разработка которого является на сегодняшний день чрезвычайно сложной задачей.
Однако, более важным этапом является привлечение и удержание клиента на веб-сайте с целью совершения сделки. Для привлечения и удержания используются различные средства, такие как красочный и удобный дизайн, использование таргетинговой рекламы, предоставление системы скидок и бонусов, использование технологий, повышающих функциональные возможности сайта.

Несмотря на все описанные выше средства и усилия, затраченные на разработку качественного веб-сайта - зачастую целевого трафика (посетителей) оказывается недостаточно, из-за чего уровень конверсий не растет или отсутствует.

На сегодняшний день одним из популярных методов борьбы с низким уровнем конверсии является прогнозирование поведения посетителей вебсайта. Существует большое количество алгоритмов, методов и методик, направленных на прогнозирование поведения, однако большая часть из них имеют проблемы, которые скрывают весь потенциал использования данных инструментов, в частности: данные, используемые для прогнозирования, являются частичными; большие объемы данных влекут за собой большую временную и пространственную сложность; методики прогнозирования являются неточными из-за недостаточной доступности пользовательских шаблона навигации.

Анализ существующих алгоритмов и подходов используемых при прогнозировании поведения пользователя позволит улучшить качество прогнозов и как следствие - увеличить конверсию вебсайта следом за которой грядет увеличение доходов веб-сайта. Кроме того, следует учесть, что почти два миллиона людей по всему миру используют социальные сети и это число увеличивается с каждым днем. Современные пользователи социальных медиа ожидают, что они смогут общаться с менеджерами, задавать вопросы и получать различные банковские услуги не закрывая окна браузера. 
Простого присутствия компании в социальных сетях уже недостаточно - все больше потребителей ожидают от банков, что тебе будут использовать социальные каналы для того, чтобы получить более быстрое, качественное и эффективное обслуживание. Пользователи социальных медиа хотят иметь доступ к персонализированным финансовым советам, финансовым предложения, а также к механизму обратной связи для банковских продуктов и услуг.

К сожалению, большинство банков не предлагают таких услуг сегодня. Среднее время ожидания ответа от представителя или менеджера в социальных сетях редко бывает меньше нескольких часов. А более чем две трети всех вопросов клиентов и вовсе остаются неотвеченными [3].

Банки не знают как использовать социальные сети в своих интересах, или не осведомлены о возможных методах взаимодействия с ними.

Тем не менее, стратегическое использование социальных медиа может значительно увеличить влияние не только на то, как финансовые учреждения продают свои услуги на рынке, но и на то, как они управляют своими рисками, как предоставляют свои услуги, занимаются анализом банковских продуктов и прогнозированием потребностей клиентов.

\section{Прогнозирование поведения посетителя веб-сайта}

В настоящее время Интернет стал неотъемлемой частью повседневной жизни, в результате чего наблюдается значительное увеличение данных, генерируемых пользователями. Этих данных огромное количество, которые также являются - неструктурированными и неупорядоченными по своей природе. Поэтому моделирование поведения и прогнозирование интереса пользователя стали очень важными задачами. Для построения моделей или проведения прогнозирования, прежде всего, необходимы данные. Процесс сбора данных - согласно выбранной предметной области - называется веб-майнингом. В процессе веб-майнинга участвуют веб-документы, гиперссылки веб-страниц, лог-файлы веб-сайтов, логи метрики веб-сайтов и т.д. Тремя основными категориями веб-майнинга являются:

1) майнинг веб-контента - процесс извлечения полезных данных из контента (текста, изображений, аудио, видео, гиперссылок и метаданных);

2) майнинг веб-структуры - процесс анализа структуры соединения веб-сайта. Для анализа применяются знания из области теории графов, например - узлами графа являются страницы, а гиперссылки - ребрами;

3) майнинг использования веб-сайта - процесс майнинга, который использует логи веб-доступа [4]. Для анализа пользовательского поведения браузинга на основе навигационного шаблона пользователя.
Данные, использующиеся в процессе вебмайнинга можно разделить на две категории - первичные данные (данные, которые доступны через сеть Интернет) и вторичные (данные лог файлов, которые могут быть использованы в процессе вебмайнинга). Прогнозирование поведения посетителя веб-сайта на основе данных, полученных в ходе вебмайнинга, может находить следующее применение:

- улучшения персонализации - возможность улучшить работу веб-сайта, выдавая пользователю результаты в соответствии с потребностями пользователя;

- улучшение качества системы - улучшение качественных показателей системы является ключевым фактором для удовлетворения потребностей пользователя. Такая возможность достигается за счет анализа сетевого трафика и метрик различных частей системы [5];

- улучшения дизайна - данное применение обусловлено наличием данных относительно того, какие части веб-сайта привлекают внимание, какие страницы наиболее посещаемы и т.д. Данная метрика, является ключевой информацией для принятия решений относительно изменений в веб-дизайне и структуре веб-сайта;

- бизнес-аналитика - полученные данные можно использовать для составления маркетинговых стратегий, принятия решений по продвижению веб-сайта, размещению рекламы и пр.

Поведение посетителя веб-сайта являет собой перечень характеристик, которые могут быть получены в результате анализа пользовательской сессии, а именно - на каких страницах оставался пользователь, какой путь для навигации между страницами использовался пользователем, почему пользователь может не достичь той или иной страницы, какие страницы привлекают больше всего внимания и т.д.

В ходе прогнозирования поведения пользователей могут использоваться различные алгоритмы и подходы, например:

1) задача о нахождении наибольшей общей подпоследовательности [6] - используется для анализа и обработки шаблонов навигации пользователя для последующего прогнозирования;

2) алгоритм анализа использования веб-сайта на основе последовательности чисел - используется для анализа сессионных шаблонов пользователя, трансформируя их в двоичную систему и затем используя стратегию поиска в глубину [7];

3) самоорганизующаяся карта Кохонена - используется в методах кластеризации;

4) модель Маркова в сочетании с алгоритмом вычисления базовой условной последовательности [8];

Использование приведенных, а также других алгоритмов, подходов и моделей позволяет повысить точность и эффективность существующих мо- 
делей. Также, при использовании известных моделей и методов прогнозирования имеет смысл:

1) прибегать к использованию гибридных схем, например метод k-средних, шаблон частого доступа и техники сопоставления схожести;

2) подготавливать данные (например - лог файлы) на стороне клиента, что позволит улучшить понимание шаблонов навигации;

3) анализировать данные, используя частые методы добычи, что позволит помочь в распознании страниц, наиболее часто посещаемых пользователем.

Комбинации различных алгоритмов для майнинга данных, построения на их основании моделей и прогнозирование поведения посетителя может значительно увеличить уровень продаж. Например одна из компании [9] в течение 2003-2004 годов использовала экспериментальную модель (модель расчета вероятности покупки) предсказания поведения пользователя. Модель помогала определить, собирается ли конкретный покупатель с высокой долей вероятности (больше 50\%) покупать некий товар n в определенный квартал. В $85 \%$ случаев покупатель действительно приобретал данный товар. Затем в модель был добавлен другой товар у, и вероятность предсказания покупки конкретным покупателем товаров n и у составляла 74 и $80 \%$ соответственно. Таким образом, данная модель увеличила способность В2В веб-сайта точно предсказывать поведение посетителя на 54\%. Ключем в предсказании поведения посетителя веб-сайта стала функция вероятности. Данная функция описывает собой вероятность $\mathrm{L}_{\mathrm{i}}$ того, что покупатель или предприятие i приобретет товар в заданное время [7]:

$$
\mathrm{L}_{\mathrm{i}}=\prod_{\mathrm{r}_{\mathrm{i}}}^{\mathrm{R}_{\mathrm{i}}}\left(\left(\prod_{\mathrm{j}=1}^{\mathrm{J}}\left(\mathrm{f}_{\mathrm{i}}(\mathrm{t}, \mathrm{j})\right)^{\delta_{\mathrm{ijt}}}\right)^{\mathrm{C}_{\mathrm{r}_{\mathrm{i}}}} \mathrm{S}_{\mathrm{i}}(\mathrm{t})^{1-\mathrm{C}_{\mathrm{r}_{\mathrm{i}}}}\right),
$$

где $\mathrm{R}_{\mathrm{i}}$ - число определяющее время между покупками покупателя или предприятия $\mathrm{i} ; \mathrm{C}_{\mathrm{r}}=0$, если время покупки находится вне наблюдаемого времени, иначе $\mathrm{C}_{\mathrm{r}}=1 ; \delta_{\mathrm{ijt}}=1$, если продукция $\mathrm{j}$ куплена покупателем или предприятием і в момент времени $t$, иначе $\delta_{\mathrm{ijt}}=0 ; \mathrm{f}_{\mathrm{i}}(\mathrm{t}, \mathrm{j})$ - плотность соответствующего распределения; $\mathrm{S}_{\mathrm{i}}(\mathrm{t})$ - функция выживаемости, она является функцией цензурирования данных, поскольку конец периода сбора данных в данном эксперименте не совпадает с периодом покупок всех предприятий. Если же привести цифры применения данной экспериментальной модели, то:

1) доход с одного посетителя веб-сайта типа В2В увеличился на 1600 у.е. (увеличение дохода веб-сайта с 200000 посетителей примерно на 320 миллионов у.е.);

2) доход с одного посетителя веб-сайта компании, занимающейся предоставлением финансовых услуг, составил 400 у.е. (увеличение дохода с клиентской базы в 10000 пользователей составляет около 4 миллионов у.е.)

\section{Анализ методов использования социальных сетей в интересах банка}

Финансовые учреждения смогут получить выгоду сразу в нескольких сферах, благодаря преимуществам, которые дают им социальные сети.

Полный отказ от использования социальных сетей приведет к стратегическому самоуничтожению в современном мире, поскольку преимуществ намного больше рисков. Первоначально, социальные банковские медиа-технологии и решения были сосредоточены на простых функциях, таких как социальный маркетинг или ответы на вопросы клиентов. Сегодня необходимо комплексное решение, которое охватывает множество возможностей, включая взаимодействие с клиентами, анализ данных, получение информации, управление рисками и т. д. Социальные данные помогут финансовым учреждениям не только увеличить уровень удовлетворенности клиента продуктами компании, но и в перспективе привлечь новых клиентов, а также увеличить долю средств клиента, которую он хранит в банке, пользуясь теми или иными банковскими услугам.

Для усиления своих конкурентных позиций нужно постоянно улучшать свои сервисные возможности путем уменьшения времени ожидания ответа. Еще один способ достичь конкурентного преимущества - введение персонализированных предложений, которые должны формироваться на основе открытых социальных данных пользователя сети.

Банки сегодня используют социальные медиа в основном для маркетинга и продвижения (табл. 1). Тем не менее, они могут расширить сферу их применения путем выбора правильных аналитических инструментов и социальных платформ. Чтобы полностью раскрыть потенциал использования социальных сетей, а не останавливаться лишь на продвижении и репутации бренда, необходимо научиться предсказывать поведение клиента, основываясь на его социальных данных.

Количество людей,

Таблица 1

подписанных на популярные украинские банки в Facebook и Twitter в ноябре 2017г.[9]

\begin{tabular}{|l|l|l|}
\hline Название банка & Facebook & Twitter \\
\hline ПАТ КБ "ПРИВАТБАНК" & 95500 & 113000 \\
\hline АО "Райффайзен Банк Аваль" & 23000 & - \\
\hline ПАТ "АЛЬФА-БАНК" & 20537 & 2600 \\
\hline ПАТ "УКРСОЦБАНК" & 13300 & 400 \\
\hline АТ "ОТП БАНК" & 18848 & 248 \\
\hline
\end{tabular}

Один из методов использования социальных сетей - получение дополнительных данных о пользователях. Для понимания всех требований современного клиента необходим унифицированный анализ данных с многих источников. Современные аналитические инструменты позволяют объединить 
внутренние банковские данные, с такими социальными данными, как например, дни рождения, годовщины или другими важные для клиента даты. Все эти данные помогут предоставить клиенту более привлекательный банковский продукт. Используя эти данные, можно также лучше управлять своими рисками, например, заблаговременно идентифицировать потенциальных неплательщиков.

Социальные медиа могут быть эффективным каналом для уведомления и разъяснения этих изменений клиенту. Новые требования регулятора или изменения в законодательстве часто изменяют условия ведения бизнеса и соответственно предоставления тех или иных банковских услуг. Пользователи также могут узнавать о нововведениях и обновлениях в продуктах и услугах банка.

Еще один метод использования социальных сетей - возможность отслеживания поведения пользователей путем мониторинга их реакции на те или иные сообщения на официальных страницах банка. Перепосты, лайки и комментарии можно использоваться для создания представления о восприятии пользователями той или иной информации о продуктах и услугах. Аналитические инструменты предоставляют неоценимую информацию о поведении клиентов и их настроениях, что можно использовать для разработки более клиенто-ориентированных и персонализированных продуктов и услуг (рис. 2).

\begin{tabular}{|c|c|c|c|}
\hline \multirow[b]{2}{*}{ VAR CODE } & \multicolumn{3}{|c|}{ ORDIYARY DEFAULT SCORECARD } \\
\hline & $\begin{array}{c}\text { Variable } \\
\text { name }\end{array}$ & Value range & Scorepoint \\
\hline & & Male & 15 \\
\hline \multirow[t]{5}{*}{$x_{1}$} & $\begin{array}{c}\text { Number of } \\
\text { days since } \\
\text { last visit }\end{array}$ & $x 1<1$ & 26 \\
\hline & & $1<=x 1<3$ & 30 \\
\hline & & $3<=x 1<37$ & 20 \\
\hline & & $37<=x 1<149$ & 17 \\
\hline & & $149<=x 1$ & 11 \\
\hline \multirow[t]{4}{*}{$x_{30}$} & $\begin{array}{l}\text { Number of } \\
\text { days since } \\
\text { the first post }\end{array}$ & $x 30<265$ & 20 \\
\hline & & $265<=\times 30<399$ & 15 \\
\hline & & $399<=x 30<1330.5$ & 23 \\
\hline & & $1330.5<=x 30$ & 36 \\
\hline \multirow[t]{2}{*}{$x_{7 s}$} & $\begin{array}{l}\text { Number of } \\
\text { job places }\end{array}$ & 0, Missing & 21 \\
\hline & & $>=1$ & 29 \\
\hline
\end{tabular}

Рис. 2. Пример системы показателей для расчета рейтинга основанного на социальных данных [10]

Социальные медиа и мобильные приложения можно использовать и в качестве виртуальных “отделений банка", что позволит увеличить проникновение в те географические области, где у них нет физического присутствия. Используя каналы социальных сетей, можно вовлечь новых клиентов, предлагая им различные услуги, такие как дебетовые или кредит- ные карты, депозиты, беспроцентные переводы в тех областях или регионах, где открывать новое отделение нецелесообразно по финансовым причинам. Каналы в социальных сетях могут содержать информацию о продуктах и услугах конкурентов. Путем отслеживания этих данных можно оценить реакцию пользователей на предложения конкурентов и понимать, какие из аспектов их стратегий можно использовать в собственных продуктах.

Отдельная команда SMM (social media marketing) может сделать банк более доступным и отзывчивым для своих клиентов. Быстрые ответы на вопросы и предоставление точной информации повысит уровень удовлетворенности и доверия клиентов.

Многие социальные сети предлагают компаниям возможность размещения приложений. Этим могут воспользоваться и банки. Этот метод увеличивает лояльность клиентов и удобство пользования продуктом. Некоторые банки уже запустили услугу привязки своей учетной записи к странице пользователя Facebook, обеспечивая таким образом быстрые денежные переводы между друзьями, удобную покупку билетов в кино для компании друзей и т.д.

Социальные сети могут широко использоваться и самими сотрудниками. Так, например, они могут делиться своим опытом, помогать друг другу решать рабочие вопросы или распространять важную внутреннюю информацию. Согласно опросам, 20\% сотрудников используют социальные сети для получения информации касательно работы, а $24 \%$ используют их для общения с коллегами [11].

К сожалению, внедрение и использование этих методов требует определенных усилий, а во время внедрения можно столкнуться с определенными трудностями. Для внедрения всех этих инструментов необходима специальная команда, которая будет управлять социальными каналами. Социальные сети должны быть внедрены в общую экосистему банка. Более того, учреждения должны разработать системy KPI (key performance indicator) для оценки своей деятельности в сфере социальный данных.

Прежде чем выбрать инструмент или платформу, предстоит определить свое видение социальной стратегии. Например, в Украине наиболее популярными социальными сетями являются Facebook и Instagram [12], делая их очевидным выбором для использования в качестве социального канала.

Возможно, придется внедрить новые метрики для измерения эффективности социальной стратегии. Эти данные могут помочь понять, кто является целевой аудиторией банка, где эти клиенты обсуждают продукты банка, и что они о них говорят. Эта информация может стать ключевой в процессе поиска способа улучшить банковский продукт. Успех в социальных медиа, тем не менее, также требует наличия хорошо развитой ИТ инфраструктуры в финансовом учреждении. При планировании деятельности стоит учитывать, что технологии должны быть на первом плане. 
Возможно для эффективного внедрения социальной стратегии многим банкам понадобится перестроить свою устоявшуюся ИТ-инфраструктуру. Технологии должны быть объединены для обеспечения единого, последовательного и полностью интегрированного обслуживания клиентов независимо от канала взаимодействия (рис. 3).

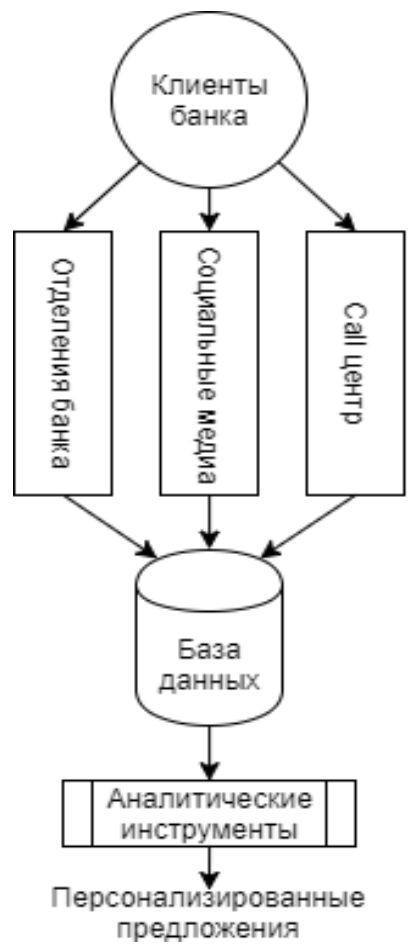

Рис. 3. Схема инфраструктуры анализа данных клиентов банка

Интегрированная ИТ-архитектура, в которой используются социальные сети, может охватывать всю гамму банковских операций, от клиентских операций фронт-офиса до обмена информацией между фронт-офисом, мидл-офисом и бэк-офисом. Это позволит эффективно коммуницировать, сокращать расходы на деятельность, не связанную с добавленной стоимостью, и больше ориентироваться на стратегическую деятельность.

Развертывание социальных CRM может стать следующей важной задачей для банков, поскольку это позволит им осуществлять мониторинг и обрабатывать большие объемы неструктурированных данных с целью автоматизации процесса создания персонализированных предложений в соответствии с индивидуальными потребностями клиента.

По сравнению с другими отраслями банки собирают данные, которые много говорят об их клиентах. К таким данным можно отнести покупательские настроения и предпочтения, выбор инвестиций, важные даты, такие как дни рождения и юбилеи. Объединяя эти данные можно получить полную поведенческую картину клиента, которая показывает потребности и желания, прогнозирует спрос на банковские продукты и услуги, при этом подстраивая их под постоянно меняющиеся запросы клиента.
При сборе и хранении персональных данных необходима осторожность. Рекомендуется установить внутренние правила и регуляции, касающиеся работы с данными клиентов. Информационная политика должна учитывать действующее законодательство, соглашения о конфиденциальности и этические аспекты ведения бизнеса.

Опрос, проведенный в Harvard Business Review, выявил, что 50\% опрошенных финансовых учреждений в настоящее время используют социальные сети, 25\% планируют использовать, а 22\% не планировали использовать социальные медиа [13]. Учитывая ожидания клиентов, последняя группа подвергает себя риску (рис. 4), и может стать неконкурентоспособной, поскольку это банк, который не планирует интегрировать социальные медиа в свои основные бизнес-операции.

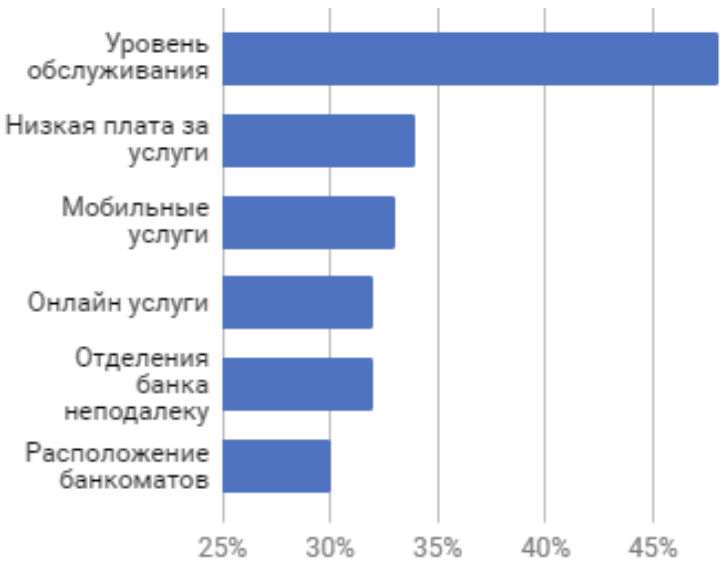

Рис. 4. Причины выбора банка согласно опросу Harris Interactive [14]

Метод проведения успешной социальной медиа-стратегии определяется в следующих шагах (с приблизительной длительностью):

- разработать видение и получить необходимую организационную поддержку для охвата аудитории социальных сетей (3 недели) 4

- определить масштаб и задачи для медиастратегии (1 неделя)4

- определить правильные метрики для измерения эффективности стратегии (1 неделя);

- разработать план надежного снижения рисков (2 недели);

- изучить и понимать регуляторные требования и требования о конфиденциальности (3 недели);

- интегрировать технологическую инфраструктуру для успешного продвижения социальной стратегии и достижения бизнес-цели (2 месяца);

- обеспечить организацию обучения корпоративной социальной стратегии в масштабах всей организации (3 месяца).

Так или иначе, нельзя отрицать, что социальные сети стали необходимыми для банковской отрасли, и вопрос не в том, нужно ли, а в том, как банкам образовать социальные стратегии для обеспечения прибыльности в будущем. 


\section{Выводы}

Интернет является стремительно растущей информационной сетью, а объемы данных вместе с количеством пользователей в сети растут колоссальными шагами. Анализ прогнозирования поведения посетителя веб-сайта, улучшение существующих алгоритмов и подходов позволит вести бизнес с гораздо большей эффективностью и получать значительное увеличение доходов. Сфера социальных медиа быстро развивается. Банки и финансовые учреждения, которые смогут быстро объединить свои бизнес-операции и стратегии в социальных сетях, будут более гибкими к потребностям клиентов и смогут предложить своим клиентам лучший опыт в использовании финансовых продуктов.

Необходимость проведения дополнительных исследований в области прогнозирования поведения посетителей веб-сайтов и социальных сетей отчетливо прослеживается с дальнейшим развитием сети интернет. Усовершенствование алгоритмов и подходов используемых при прогнозировании поведения посетителей веб-сайта и социальных сетей позволят пользователям более эффективно и комфортно осуществлять действия на веб-сайте, такие как поиск информации, осуществление покупок, общение в социальных сетях и т.д. Бизнес, в свою очередь, будет получать увеличенную конверсию пользователей, увеличенные доходы которые позволят осваивать новые рынки сбыта, помогая достигать максимальной коммерческой выгоды.

\section{Список литературы}

1. Понятие электронной коммеричи [Электронный ресурс] // Режим доступа к статье: $\underline{\text { http }}$ http://laboureconomics.ru/neweconomics/131-ecommerce.

2. US e-commenrce sales grow 15.6\% in 2016 [Электронный ресурс] // Режим доступа $\kappa$ статье: https://www.digitalcommerce360.com/2017/02/17/.

1. The Wall Street Journal, March 13, 2014, [Электронный ресурс] // Режим доступа $\kappa$ cтатье: http://online.wsj.com/news/articles.
3. Sudheer K. Reddy. An Effective Methodology for Pattern Discovery in Web Usage Mining, International Journal of Computer Science and Information Technologies, Vol. 3 (2) 2012, pp. 3664-3667.

4. Vedpriya Dongre, Jadgish Raikwal, An Improved user Browsing Behavior Prediction Using Web Log Analysis, Internmational Journal of Advanced Research in Computer Engineering \& Technology, Volum 4 Issue 5, May 2015, pp. $1838-1842 c$.

5. Наибольшая общая подпоследовательность [Электронный ресурс] // Режим доступа: https://en. wikipedia.org/wiki/Longest common subsequence problem.

6. Gang Fang, Jia-Le Wang, Hong Ying, Jiang Xiong, $A$ double algorithm of Web usage mining based on sequence number, IEEE 2009

7. A. Awad and Issa Khalil, Prediction of User's WebBrowsing Behavior: Application of Markov Model, IEEE Transaction 2010.

8. Knowing What to Sell, When, and to Whom [Электронный ресурс] // Режим доступа: https://hbr.org/ 2006/03/knowing-what-to-sell-when-and-to-whom.

9. The Wall Street Journal, March 13, 2014, ГЭлектронный ресурс] // Режим доступа $\kappa$ статье: http://online.wsj.com/news/a.rticles/SB1000142405270230354 6204579437692833009398

10. Офиџиальный рейтинг банков Украины [Электронный ресурс] // Режим доступа $\kappa$ статье: http://banker.ua/officialrating/.

11. CREDIT SCORING BASED ON SOCIAL NETWORK DATA [Электронный ресурс] // Режим доступа: https://bijournal.hse.ru/data/2015/10/12/1076342406/2.pdf.

12. Social Media and the Workplace // Режим docmyna к cmambe: http://www.pewinternet.org/2016/06/22/ social-media-and-the-workplacel

13. Рейтинг популярных в Украине сайтов за октябрь 2017 года [Электронный ресурс] // https://itc.ua/ news/reyting-populyarnyih-v-ukraine-saytov-v-oktyabre-2017goda-sotsialnyie-seti-novostnyie-i-e-commerce-saytyi-vyiroslia-sanktsionnyie-resursyi-poteryali-ohvat/.

14. Harvard Business Review, 2010 [Электронный ресурс] // Режим доступа:

http://www.sas.com/resources/whitepaper/wp_23348.pdf.

Надійшла до редколегії 22.12.2017

Рецензент: д-р техн. наук, проф. К.С. Козелкова, Державний університет телекомунікацій, Київ.

\title{
АНАЛІЗ ПРОГНОЗУВАННЯ ПОВЕДІНКИ ВІДВІДУВАЧА ВЕБ-САЙТУ
}

\author{
Д.В. Гриньов, Д.С. Бойко, М.А. Голуб
}

В статті розглянуті основні підходи процесу прогнозування поведінки відвідувача веб-сайту. Наведені приклади існуючих методів та методик прогнозування, надані висновки щодо необхідності аналізу існуючих та побудови нових моделей поведінки, а також складання прогнозів за допомогою иих моделей у процесі ведення бізнесу. Також надан методи використання соиіальних мереж для збільшення числа клієнтів фінансових установ, підвищення задоволеності продуктами банку та зростання їх конкурентоспроможності. Визначається підхід до стратегії використання соиіальних медіа в банківській сфері.

Ключові слова: електронна комериія, моделі поведінки, сочіальна мережа, банк, маркетинг.

\section{BEHAVIOUR PREDICTION ANALYSIS OF WEBSITE USER}

D.V. Grynov, D.S. Boiko, M.A. Holub

The article considers the main approaches of the process of forecasting the behavior of the web site's user. Examples of existing approaches and methods of forecasting are presented as well as the conclusions regarding the need to analyze existing and building new behavioral models as well as make forecasts using these models in the course of doing business. The article describes methods of using social networks used to increase the number of clients of financial institutions, as well as to increase satisfaction with the products of the bank and increasing their competitiveness. The article also determines the approach to the strategy of using social media in the banking sector.

Keywords: e-commerce, behavior models, social network, bank, marketing. 